\title{
A cluster-randomized trial to reduce caesarean delivery rates in Quebec: cost- effectiveness analysis
}

\author{
Mira Johri ${ }^{1,2,3^{*}}$ (D), Edmond S. W. Ng ${ }^{4}$, Clara Bermudez-Tamayo ${ }^{5,6,7}$, Jeffrey S. Hoch ${ }^{8,9,10}$, Thierry Ducruet ${ }^{11}$
} and Nils Chaillet ${ }^{12}$

\begin{abstract}
Background: Widespread increases in caesarean section (CS) rates have sparked concerns about risks to mothers and infants and rising healthcare costs. A multicentre, two-arm, cluster-randomized trial in Quebec, Canada assessed whether an audit and feedback intervention targeting health professionals would reduce CS rates for pregnant women compared to usual care, and concluded that it reduced CS rates without adverse effects on maternal or neonatal health. The effect was statistically significant but clinically small. We assessed cost-effectiveness to inform scale-up decisions.
\end{abstract}

Methods: A prospective economic evaluation was undertaken using individual patient data from the Quality of Care, Obstetrics Risk Management, and Mode of Delivery (QUARISMA) trial (April 2008 to October 2011). Analyses took a healthcare payer perspective. The time horizon captured hospital-based costs and clinical events for mothers and neonates from labour onset to 3 months postpartum. Resource use was identified and measured from patient charts and valued using standardized government sources. We estimated the changes in CS rates and costs for the intervention group (versus controls) between the baseline and post-intervention periods. We examined heterogeneity between clinical subgroups of high-risk versus low-risk pregnancies and estimated the joint uncertainty in cost-effectiveness over 20,000 trial simulations. We decomposed costs to identify drivers of change.

Results: The intervention group experienced per-patient reductions of 0.005 CS (95\% confidence interval (Cl): -0 . 015 to $0.004, P=0.09)$ and $\$ 180$ (95\% Cl: $-\$ 277$ to $-\$ 83, P<0.001)$. Women with low-risk pregnancies experienced statistically significant reductions in CS rates and costs; changes for the high-risk subgroup were not significant. The intervention was "dominant" (effective in reducing CS and less costly than usual care) in $86.08 \%$ of simulations. It reduced costs in $99.99 \%$ of simulations. Cost reductions were driven by lower rates of neonatal complications in the intervention group $(-\$ 190,95 \% \mathrm{Cl}$ : $-\$ 255$ to $-\$ 125, P<0.001)$. Given 88,000 annual provincial births, a similar intervention could save $\$ 15.8$ million (range: $\$ 7.3$ to $\$ 24.4$ million) in Quebec annually.

Conclusions: From a healthcare payer perspective, a multifaceted intervention involving audits and feedback resulted in a small reduction in caesarean deliveries and important cost savings. Cost reductions are consistent with improved quality of care in intervention group hospitals.

Trial registration: International Clinical Trials Registry Platform, ISRCTN95086407. Registered on 23 October 2007

Keywords: Randomized controlled trial, Cost-benefit analysis, Caesarean section/utilization, Pregnancy outcomes, Medical audit, Guideline adherence, Multilevel analysis, Female, Adult, Adolescent, Infant, Newborn

\footnotetext{
* Correspondence: mira.johri@umontreal.ca

${ }^{1}$ Centre de Recherche du Centre Hospitalier de l'Université de Montréal

(CRCHUM), Tour Saint-Antoine, Porte S03-910, 850, rue St-Denis, Montréal,

Québec H2X 0A9, Canada

${ }^{2}$ Department of Health Management, Evaluation and Policy, School of Public

Health, University of Montreal, Montréal, Québec, Canada

Full list of author information is available at the end of the article
}

(c) The Author(s). 2017 Open Access This article is distributed under the terms of the Creative Commons Attribution 4.0 International License (http://creativecommons.org/licenses/by/4.0/), which permits unrestricted use, distribution, and reproduction in any medium, provided you give appropriate credit to the original author(s) and the source, provide a link to the Creative Commons license, and indicate if changes were made. The Creative Commons Public Domain Dedication waiver (http://creativecommons.org/publicdomain/zero/1.0/) applies to the data made available in this article, unless otherwise stated. 


\section{Background}

The use of caesarean sections has increased to unprecedented levels worldwide; $18.6 \%$ of global and $32.3 \%$ of North American births now occur by caesarean section [1]. With the exception of the African region, where caesarean section rates remain low, substantial increases in caesarean deliveries have been documented in all global regions [1]. Caesarean sections are important in reducing maternal and perinatal mortality and morbidity when medically justified; however, non-medically necessary caesareans have no documented benefit [2]. In many jurisdictions, caesarean section rates in excess of recommended thresholds have sparked concerns about potential risks to mothers and infants and escalating healthcare costs $[1,3,4]$. Evidence concerning effective approaches to reduce unnecessary caesareans is currently limited $[3,5]$.

The Quality of Care, Obstetrics Risk Management, and Mode of Delivery (QUARISMA) study was a cluster-randomized controlled trial conducted from 2008 to 2011 in 32 hospitals in Quebec, Canada [6]. The trial assessed whether a multifaceted audit and feedback intervention targeting health professionals involved in labour, delivery, and postpartum care would reduce caesarean section rates as compared to usual care for pregnant women [6]. The idea motivating the QUARISMA trial was that an effective knowledge translation intervention could improve adherence to clinical practice guidelines and quality of care, thereby reducing unnecessary caesareans and optimizing resource use.

The main trial analysis found a statistically significant but clinically small reduction in caesarean sections (adjusted absolute risk difference of $-1.8 \%$ ( $95 \% \mathrm{CI}:-3.8$ to -0.2 , $P=0.04$ ) with no adverse effects on maternal or neonatal health outcomes [6]. The reduction in caesareans was observed among women with low-risk pregnancies but not among women with high-risk pregnancies [6]. Although resources required to deliver the intervention are modest, the anticipated magnitude of clinical benefit is small, leaving unanswered questions about whether the intervention should be offered at scale. A cost-effectiveness analysis is required to complete information from the trial and enable decision makers to interpret results for policy and practice.

We conducted a cost-effectiveness analysis of the QUARISMA trial using individual patient data. Our primary objective was to compare the impact on caesarean delivery rates and costs of a multifaceted audit and feedback intervention targeting health professionals versus usual care for pregnant women. Subgroup analyses examined costeffectiveness in high-risk versus low-risk pregnancies, the clinical subgroups established a priori for the trial.

\section{Methods}

The QUARISMA trial was conducted in the province of Quebec, Canada. In 2014, Quebec's population was 8.2 million, and life expectancy at birth was 79 for males and 83 for females [7]. Gross domestic product per capita measured in US dollar purchasing power parity was $\$ 36,216$ in 2013 [8]. The QUARISMA economic evaluation adopted the perspective of the publicly funded healthcare system in Quebec. Analysis of effects focused on the primary hypothesized trial outcome of reduction in caesarean sections, while cost analysis examined direct costs to the public healthcare system. Cost variables capture the full spectrum of resource use related to labour and delivery from hospital admission for labour until 3 months postpartum. As delivery mode (caesarean or vaginal) was the primary trial endpoint, a short-term hospital stay is sufficient to capture all meaningful differences in costs and clinical outcomes between intervention and control arms. Costs and health outcomes were discounted at an annual rate of $0 \%$ due to the short $(<1$ year) period for results assessment $[9,10]$.

All participating hospital centres granted research ethics approval for the trial.

\section{Trial overview}

Details of the QUARISMA trial have been reported [6]. Briefly, QUARISMA employed a cluster-randomized design with a 1:1 allocation ratio. Clusters were 32 public hospitals in the province of Quebec, Canada. Hospitals were stratified by level of care and assigned to either the intervention or control group using computer-generated blocked randomization within each stratum. Hospital centres were eligible to participate if they had at least 300 deliveries in the year prior to study initiation, a caesarean section rate of at least $17 \%$, and no concurrent program to reduce caesareans. Based on these criteria, 40 hospitals were eligible, 38 agreed to participate, and 32 were randomly selected for inclusion. All women who gave birth at a participating hospital during the study period and whose newborns met criteria related to gestational age ( $\geq 24$ weeks) and birth weight $(\geq 500 \mathrm{~g})$ were included in the analysis.

The study comprised three phases: a 1-year preintervention (baseline) period, a 1.5-year intervention period, and a 1-year post-intervention period. The baseline period involved onsite training and capacity building to improve caesarean delivery and intrapartum care. During the 1.5-year intervention period, hospital audit committees implemented four 3-month audit cycles using local data to assess the appropriateness of caesarean delivery, engage in collective learning, provide feedback to clinicians, and implement best practices based on the results. Analyses for the main trial and the economic evaluation compared outcomes in the 1-year baseline period (1 April 2008 to 31 March 2009) to those in the 1-year post-intervention period (1 November 2010 to 31 October 2011). The QUARISMA trial captured 
more than $65 \%$ of all deliveries in Quebec province during the study period [11]. No hospital or woman was lost to follow-up.

\section{Sample size}

QUARISMA was designed and powered to detect differences between treatment groups in the primary clinical endpoint (caesarean sections averted). Sample size calculations were not designed to test cost-effectiveness hypotheses.

\section{Effects}

We recorded clinical events from patient charts. Trained data collectors extracted data concerning caesarean or vaginal delivery as well as secondary outcomes including major and minor maternal complications and major and minor neonatal complications [6]. Trained research nurses or medical archivists abstracted in-hospital data from the medical records of mothers and newborns 3 months after delivery. Data collectors were aware of randomization assignments but were not involved in outcomes assessment. Given the nature of the clinical condition and the short time horizon, we did not include preference-based measures of health-related quality of life.

\section{Resource use and costs}

We considered resource use and costs associated with delivery and complications recorded in the QUARISMA trial (Additional file 1: Section 3; Table S1) [6]. Medical procedure costs (Additional file 1: Table S2 and Table S3) were calculated as the sum of inpatient (hospital) costs and physician fees. For all trial participants contributing data to either the baseline or post-intervention period, we used inpatient chart data to identify clinical events generating resource use. To estimate inpatient procedure costs, we applied unit costs from the 2013 Canadian Institute for Health Information (CIHI) Patient Cost Estimator (PCE) for the jurisdiction of Quebec to resource use categories [12]. Published annually by the Canadian government, PCE costs represent the product of the costs of a standard hospital stay in a specific jurisdiction multiplied by a resource intensity weight reflecting resource use for a specific case mix and age group [12]. The 2013 PCE release includes 2010-2011 financial information [12], which corresponds to the QUARISMA post-intervention period. PCE costs exclude physician fees, which were taken from Canada's National Physician Database (NPD), 2011-2012 [13]. Total costs also included resource requirements and costs associated with delivery of the QUARISMA intervention (Additional file 1: Table S4), which were estimated directly from QUARISMA trial records. Protocol-driven costs were excluded [9]. All costs are reported in 2013 Canadian dollars. In
2013, one US dollar was worth on average 1.03 Canadian dollars [14].

\section{Statistical analysis}

Analyses for the QUARISMA economic evaluation were based on the intention-to-treat principle and carried forward strategies from the main trial analysis [6]. We adopted a variant of the difference-in-differences approach to estimate the intervention effect while controlling for unobserved characteristics of individual patients or program placement in hospitals that might lead to selection bias [15]. Specifically, to assess intervention impact, we studied changes in caesarean delivery rates and costs in the two study groups between the 1-year baseline period and the 1-year post-intervention period using adjusted regression coefficients (with their 95\% confidence intervals) for the interaction between group (intervention or control) and time period (post-intervention or baseline) $[6,16]$.

\section{Main analysis}

We modelled costs and effects jointly using bivariate multilevel linear models that explicitly recognize potential correlations between the bivariate outcomes (caesarean sections and costs) at individual and cluster (hospital) levels. In our models, individual patients (level 1) were assumed to be nested within hospitals (level 2), and correlations were estimated at both hierarchical levels. We used cluster-level random effects to handle clustering of observations within hospitals $[17,18]$. The analysis included all trial participants. Crude models studied the intervention effect by estimating the interaction between study group and time period. Adjusted models estimated the same interaction term while including additional baseline covariates (pregnancy risk, parity, current smoking, birth weight, and hospital type) used in the QUARISMA trial analysis [6]. Models were estimated by restricted unbiased iterative generalized least-squares estimation implemented in MLwiN (version 2.35) [19] within Stata 14.1 using the runmlwin [20] command.

We had intended to calculate the incremental costeffectiveness ratio (ICER) statistic as the ratio of additional costs per additional caesarean sections averted. However, interpretation is problematic when ICER estimates are negative, as in this case where the intervention reduces costs [21]. Incremental costs and effects are therefore presented separately.

\section{Uncertainty and heterogeneity of the cost-effectiveness measures}

We applied Bayesian Markov chain Monte Carlo methods to the bivariate multilevel linear models used in the main analysis to ascertain the joint uncertainty of the estimands, incremental costs, and effects [22]. The 
posterior distributions of the incremental costs and effects were given by 20,000 iterations of the Markov chains after a burn-in of 5000 iterations. We used cost-effectiveness planes plotting the values of incremental costs and effects stored in the Markov chains to present the joint uncertainty of the estimands. To explore potential heterogeneity in the results, we prospectively planned to repeat all analyses over patient risk subgroups [21].

\section{Other analyses}

We adapted the bivariate multilevel linear models from the main analysis to consider two further issues. (1) NPD estimates for physician billings are uncertain [13] and can substantially influence total costs. We prospectively planned sensitivity analyses using alternative physician fee estimates from the Quebec medical specialists billing manual [23]. (2) To gain insight into cost drivers, we decomposed total costs into costs associated with delivery, maternal complications, and neonatal complications. Costs were grouped into these three categories because statistical power was insufficient to examine cost differences on a per-complication basis. Analyses of cost components were empirically motivated.

An independent team not involved in the main trial analysis conducted the economic analysis. All reported $P$ values are two-sided. $P$ values lower than 0.05 were considered statistically significant. Additional file 1: Section 2 expands on the statistical analysis; Tables S8 and S9 describe data clustering.

\section{Results}

\section{Participants}

All (100\%) of the 105,351 women who delivered in the baseline or post-intervention period were included in the QUARISMA main trial analysis [6] and the economic evaluation. Analysis of baseline hospital, cost, and patient characteristics (Additional file 1: Table S5) revealed no significant differences between study groups, with the exception of maternal parity, which was included as a covariate in the adjusted analyses.

\section{Incremental costs and effects}

Table 1 presents main analysis results concerning the incremental change in effects and costs due to the QUARISMA intervention. Analyses including all patients showed a small non-significant reduction in caesareans in the intervention group as compared to controls and an important reduction in costs, yielding adjusted estimates (Table 1, Model 2) of a per-patient reduction of 0.005 caesarean sections (95\% confidence interval (CI): -0.015 to $0.004, P=0.09)$ and $\$ 180$ saved (95\% CI: $-\$ 277$ to $-\$ 83, P<0.001)$. Patterns differed by patient risk. Women with low-risk pregnancies experienced larger, statistically significant reductions in caesarean sections and costs. Women with high-risk pregnancies experienced a small, non-significant increase in caesarean sections and a non-significant reduction in costs. Sensitivity analyses using alternative cost estimates predicted lower estimated savings but similar conclusions (Additional file 1: Table S6).

\section{Uncertainty of incremental costs and effects}

Figures 1 and 2 present the joint uncertainties in cost and effectiveness estimates from the Bayesian Markov chain Monte Carlo analyses on the cost-effectiveness plane. Analyses including all patients (Fig. 1) revealed that the intervention reduced caesarean sections and costs in $86.01 \%$ of the Monte Carlo iterations, and reduced costs in $99.99 \%$ of iterations. For women with low-risk (high-risk) pregnancies (Fig. 2), the intervention reduced caesarean sections in $99.81 \%(15.97 \%)$ of the iterations, reduced costs in $99.98 \%$ (90.36\%), and reduced both outcomes in $99.79 \%$ (15.24\%). Sensitivity analyses using alternative cost estimates generated similar results (Additional file 1: Figure S1 and Figure S2).

\section{Cost subcategories}

Table 2 presents changes in per-patient costs by subcategories of delivery costs, maternal complications, and neonatal complications. Findings suggest that the intervention to reduce caesarean sections resulted in improved quality along the continuum of care. Adjusted analyses including all patients revealed that the major driver of cost reductions was management of neonatal complications ( $-\$ 190,95 \% \mathrm{CI}:-\$ 255$ to $-\$ 125, P<0.001)$, which were less frequent in the intervention group [6]. Reductions in management costs for neonatal cardiopulmonary complications were especially important $(-\$ 150$, 95\% CI: $-\$ 197$ to $-\$ 103, P<0.001)$; costs for other neonatal complications were also reduced ( $-\$ 41,95 \% \mathrm{CI}$ : $-\$ 80$ to $-\$ 1, P<0.047)$. Results using alternative cost assumptions were similar (Additional file 1: Table S7).

\section{Discussion}

The QUARISMA trial found that an intervention involving clinical audits, feedback, and implementation of best practices resulted in a statistically significant but small reduction in caesarean sections without adverse effects on maternal or neonatal outcomes [6]. In this economic evaluation conducted alongside the main trial, the analysis including all trial participants found that the intervention also conferred significant average cost savings of \$180 (95\% CI: \$83-277) per patient, equivalent to $3.0 \%$ (95\% CI: 1.4-4.5\%) of mean per-patient total costs. Patterns differed by patient risk. Women with low-risk pregnancies experienced statistically significant reductions in caesarean sections and costs, while women with high-risk pregnancies did not experience significant changes in either outcome. 
Table 1 Impact of the QUARISMA intervention on caesarean sections and total direct medical costsa, b

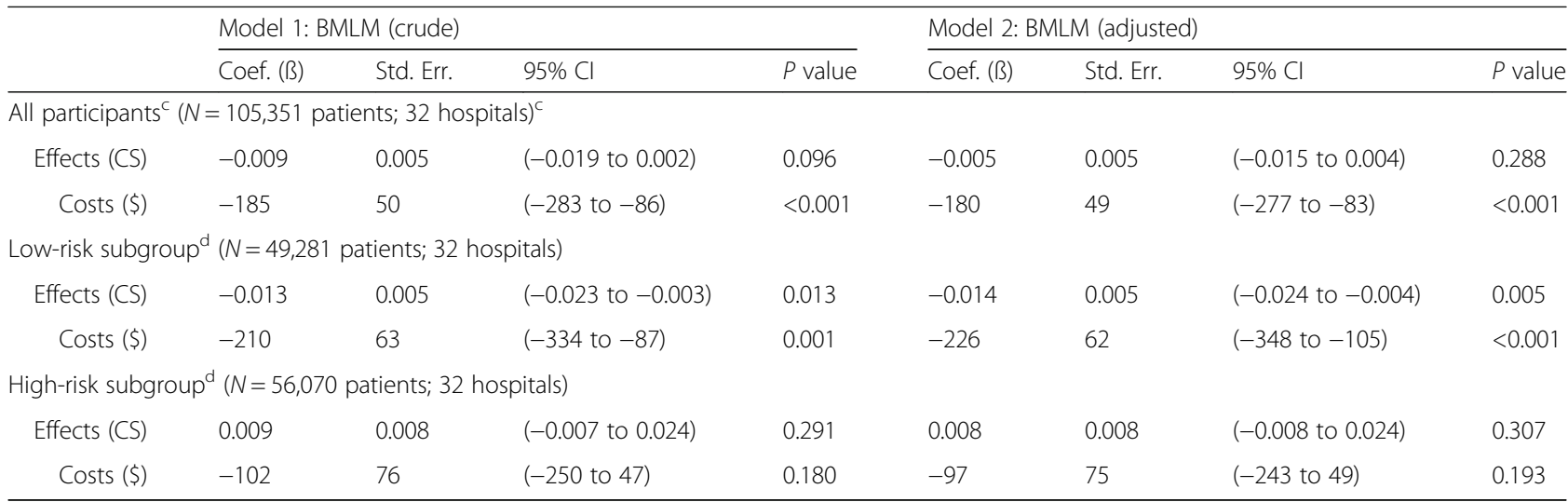

$B M L M$ bivariate multilevel linear model, $C S$ caesarean section

${ }^{a}$ All costs given in 2013 Canadian dollars = (0.94 USD) [30]

${ }^{\text {b}}$ Total costs calculated using CIHI National Physician Database 2011-2012 physician fees for the Province of Quebec [13]

'Adjusted models for all participants included the following covariates: parity, smoking, birth weight, hospital type, and women's risk level

${ }^{\mathrm{d}}$ Adjusted subgroup models included the following covariates: parity, smoking, birth weight, and hospital type

Sensitivity analyses exploring uncertainty in costeffectiveness estimates over 20,000 trial simulations demonstrated that QUARISMA was the "dominant" intervention (effective in reducing caesarean sections and less costly than usual care) $86.08 \%$ of the time. The intervention reduced costs $99.99 \%$ of the time. Cost savings largely reflect the reduced costs of managing neonatal complications, which occurred less frequently in the intervention group [6].

Our findings are important for clinicians and policymakers interested in the care of pregnant women and neonates. The QUARISMA trial tested a multifaceted strategy involving audits and feedback to enable groups of health professionals to improve the quality of labour and delivery care at participating hospitals. In addition to achieving a statistically significant but clinically small benefit in the primary outcome of reducing caesarean sections, QUARISMA also demonstrated benefits for a variety of secondary clinical endpoints consistent with improvements in the standard of care implemented in intervention group hospitals [6]. Secondary trial outcomes revealing statistically significant improvements

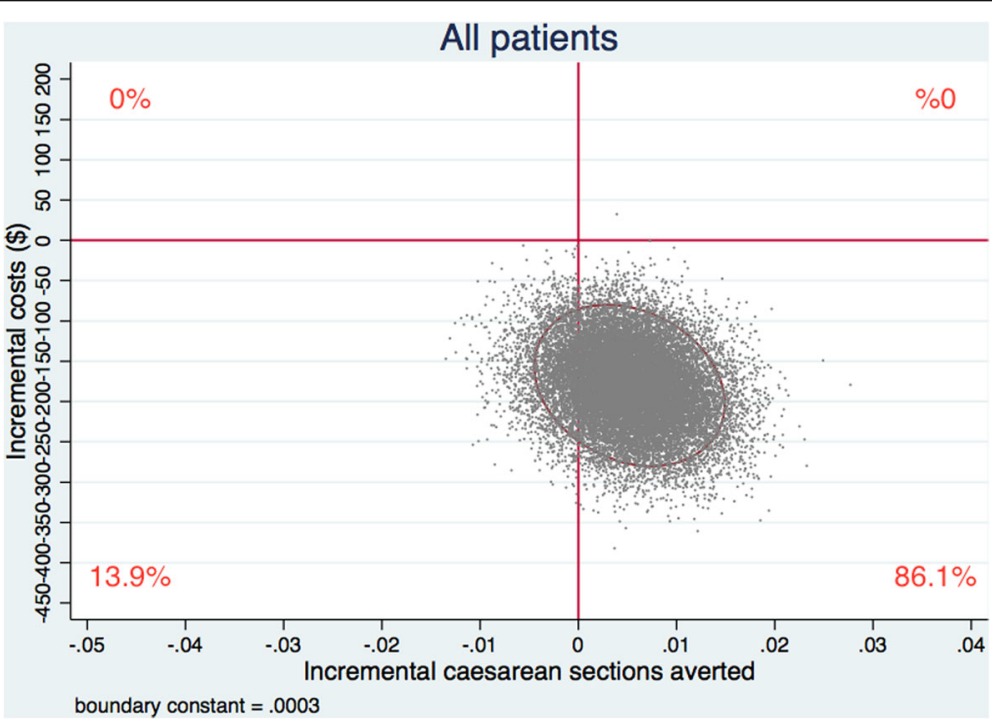

Fig. 1 Incremental cost-effectiveness of the QUARISMA intervention versus routine care. Cost-effectiveness (CE) plane for the covariate-adjusted cost-effectiveness analysis of the QUARISMA intervention versus routine care. Incremental cost-effectiveness results were based on 20,000 Markov chain Monte Carlo iterations including all trial participants ( $N=105,351)$. An ellipse containing $95 \%$ of the joint posterior distribution of incremental costs and effects is used to represent uncertainty on the CE plane. The centre of the ellipse represents the point estimate of incremental effects and costs, i.e. a per-patient reduction of 0.005 caesarean sections and $\$ 180$ saved. Percentages represent the distribution of points by quadrant 

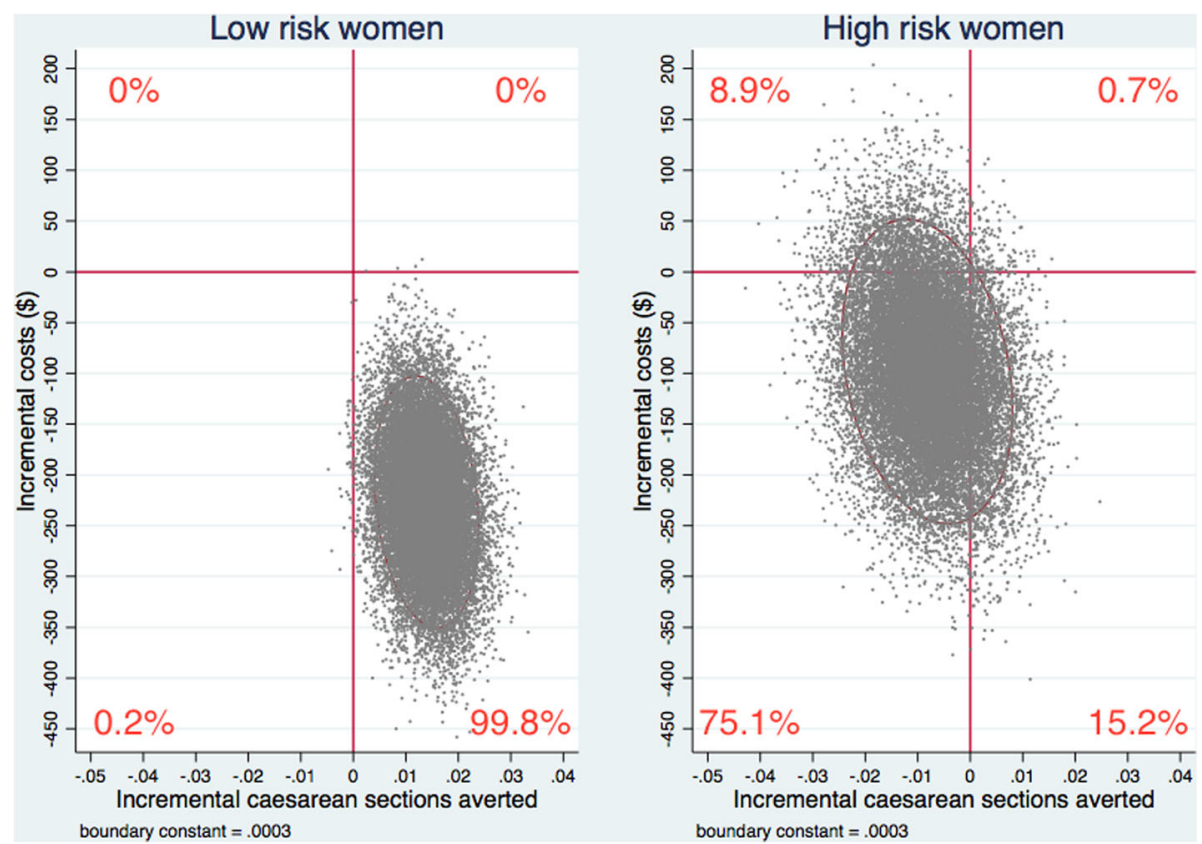

Fig. 2 Incremental cost-effectiveness of the QUARISMA intervention versus routine care, by risk subgroups. Cost-effectiveness (CE) planes for the covariate-adjusted cost-effectiveness analysis of the QUARISMA intervention versus routine care, by patient risk subgroups. Incremental cost-effectiveness results were based on 20,000 Markov chain Monte Carlo iterations estimated separately for low risk $(N=49,281)$ and high-risk $(N=56,070)$ trial participants. An ellipse containing $95 \%$ of the joint posterior distribution of incremental costs and effects is used to represent uncertainty on the CE plane. The centre of the ellipse represents the point estimate of incremental effects and costs, i.e. a per-patient reduction of 0.014 caesarean sections and $\$ 226$ saved for the low-risk subgroup, and a per-patient increase of 0.008 caesarean sections and $\$ 75$ expenditure for the high-risk subgroup. Percentages represent the distribution of points by quadrant; figures may not sum to 100 due to rounding

included minor neonatal morbidity (i.e. cardiopulmonary morbidity, moderate acidosis ( $\mathrm{pH} \geq 7$ and $<7.1$ ), minor trauma, and neonatal infection/sepsis) and major neonatal morbidity (i.e. major trauma, use of invasive mechanical ventilation, and intrapartum and neonatal deaths) [6]. Economic evaluation offers a framework within which complex changes can be synthesized to aid in policymaking. Our findings (Table 1) suggest that if a similar intervention were to be delivered at scale in the Province of Quebec, for an annual provincial birth cohort of approximately 88,000 [11] and a per-patient reduction in caesareans of 0.005 (95\% CI: -0.015 to 0.004 ), one could anticipate a reduction of roughly 440 (1320 to 0$)$ caesarean sections. For the same annual birth cohort of 88,000 [11] and a per-patient reduction in costs of $\$ 180$ (95\% CI: $-\$ 277$ to $-\$ 83$ ), this translates to a cost savings of $\$ 15.8$ million (range: $\$ 7.3$ to $\$ 24.4$ million), achieved without increasing maternal and neonatal morbidity and mortality.

These results have important implications for the scientific literatures evaluating audit and feedback approaches for quality improvement and non-clinical interventions to reduce caesarean sections. The audit and feedback strategy is widely used to promote quality improvement. A recent Cochrane systematic review found that audit and feedback generally leads to small but potentially important improvements in professional practice [24], which coheres well with the QUARISMA trial finding that the intervention conferred a modest reduction in caesarean sections [6]. Intervention impact on healthcare costs is often an outcome of central interest for quality improvement interventions. Yet, we found only one economic evaluation of an audit and feedback intervention based on data from a randomized trial, published by Fretheim and colleagues in 2006 [25]. This study is a well-conducted evaluation of a small, individually randomized trial in Norway, which found potential cost savings due to the intervention [25]. Analytic methods have advanced considerably in the interim; notably, the Fretheim and colleagues study was not based on individual patient data.

Important studies of non-clinical interventions to reduce unnecessary caesareans exist [5]; however, only one has published an economic evaluation [26]. Hollinghurst and colleagues conducted a cost-consequences analysis of a small, individually randomized trial of 742 pregnant women in the UK with a prior caesarean section [26], to evaluate two computer-based decision aids to reduce repeat caesarean sections [27]. One decision aid reduced repeat caesareans and was likely cost-neutral [27].

Ours is the first economic evaluation of a non-clinical intervention to reduce caesarean section rates based on a cluster-randomized trial. The QUARISMA trial was 
Table 2 Changes in per-patient costs due to the intervention, by clinical category ${ }^{a}$ b

\begin{tabular}{|c|c|c|c|c|c|c|c|c|}
\hline \multirow[t]{2}{*}{ Cost component } & \multicolumn{4}{|c|}{ BMLM (crude) } & \multicolumn{4}{|c|}{ BMLM (adjusted) } \\
\hline & Coef. (ß) (\$) & Std. Err. (\$) & $95 \% \mathrm{Cl}(\$)$ & $P$ value & Coef. (ß) (\$) & Std. Err. (\$) & $95 \% \mathrm{Cl}(\$)$ & $P$ value \\
\hline \multicolumn{9}{|c|}{ All participants ( $N=105,351$ patients; 32 hospitals) } \\
\hline Delivery (caesarean or vaginal) ${ }^{d}$ & -8.3 & 8.2 & $(-24.4$ to 7.7$)$ & 0.310 & -6.2 & 7.7 & $(-21.3$ to 9.0$)$ & 0.424 \\
\hline Maternal complications ${ }^{\mathrm{e}}$ & 19.9 & 30.0 & $(-38.9$ to 78.8$)$ & 0.507 & 16.3 & 29.9 & $(-42.3$ to 74.8$)$ & 0.586 \\
\hline Neonatal complications ${ }^{f}$ & -196 & 33.8 & $(-262$ to -130$)$ & $<0.001$ & -190 & 33.3 & $(-255$ to -125$)$ & $<0.001$ \\
\hline \multicolumn{9}{|c|}{ Low-risk group ( $N=49,281$ patients; 32 hospitals) } \\
\hline Delivery (caesarean or vaginal) ${ }^{d}$ & -17.2 & 9.4 & $(-35.7$ to 1.3$)$ & 0.069 & -22.0 & 9.0 & $(-39.5$ to -4.5$)$ & 0.014 \\
\hline Maternal complications ${ }^{e}$ & 22.0 & 38.4 & $(-53.2$ to 97.2$)$ & 0.566 & 14.7 & 38.1 & $(-59.9$ to 89.3$)$ & 0.700 \\
\hline Neonatal complications ${ }^{f}$ & -215 & 42.0 & $(-297$ to -133$)$ & $<0.001$ & -219 & 41.8 & $(-301$ to -137$)$ & $<0.001$ \\
\hline \multicolumn{9}{|c|}{ High-risk group ( $N=56,070$ patients; 32 hospitals) } \\
\hline Delivery (caesarean or vaginal) ${ }^{d}$ & 17.6 & 12.2 & $(-6.3$ to 41.6$)$ & 0.149 & 14.8 & 12.2 & $(-9.0$ to 38.7$)$ & 0.223 \\
\hline Maternal complications ${ }^{e}$ & 17.6 & 45.3 & $(-71.2$ to 106$)$ & 0.698 & 14.2 & 45.1 & $(-74.3$ to 103$)$ & 0.753 \\
\hline Neonatal complications ${ }^{f}$ & -136 & 51.2 & $(-237$ to -36.0$)$ & 0.008 & -126 & 50.0 & $(-224$ to -27.6$)$ & 0.012 \\
\hline
\end{tabular}

$B M L M$ bivariate multilevel linear model, $C l$ confidence interval

${ }^{a}$ Model coefficients ( $(3)$, standard errors, and 95\% confidence intervals present costs given in 2013 Canadian dollars $=(0.94$ USD) [30]

${ }^{\mathrm{b}}$ Total costs calculated using CIHI National Physician Database 2011-2012 physician fees for the Province of Quebec [13]

'The $P$ value is the probability of observing a result at least as extreme as the $z$-statistic, for the null hypothesis that the difference in the rate changes in costs for the intervention group from baseline to post-intervention versus the control group from baseline to post-intervention does not differ from zero

${ }^{\mathrm{d} D e l i v e r y ~ c o s t s ~ i n c l u d e: ~ c a e s a r e a n ~ d e l i v e r y ~(p r i m a r y ~ o r ~ s e c o n d a r y, ~ w i t h ~ o r ~ w i t h o u t ~ i n d u c t i o n, ~ w i t h ~ o r ~ w i t h o u t ~ u t e r i n e ~ s c a r, ~ p e d i a t r i c ~ o r ~ a d u l t) ; ~ v a g i n a l ~ d e l i v e r y ~(w i t h ~}$ or without anaesthetic, with or without intervention, assisted or unassisted, pediatric or adult)

e Maternal complication costs include: maternal death, hysterectomy, symptomatic uterine rupture, thromboembolic disease, internal organ injuries, perineal tear (grades 3-4), puerperal infection/sepsis, postpartum hospital stay $\geq 7$ days, admission to intensive care unit, readmission to hospital after postpartum discharge, blood transfusion

fNeonatal complication costs include: intrapartum/neonatal death, Apgar score $(<4 ; 4-7)$, major and minor acidosis $(\mathrm{pH}<7 ; \mathrm{pH} 7-7.1)$, major and minor trauma, intraventricular haemorrhage, seizure at less than $24 \mathrm{~h}$, invasive and non-invasive mechanical ventilation, necrotizing enterocolitis, hypoxic-ischaemic encephalopathy cardiopulmonary morbidity, neonatal infection/sepsis, blood transfusion

well designed and implemented and captured $65 \%$ of provincial births occurring in the trial period, plausibly representing the general population of pregnant women in Quebec province. Prospective data collection improved data completeness, accuracy, and coherence, while the use of standardized government sources for costing enhanced generalizability. The economic analysis was based on the intention-to-treat principle; it used complete data from individual patients and appropriate statistical methods that accounted for clustering and correlated outcomes to derive methodologically robust estimates of between-group differences in costs and effects.

Several limitations should be considered. (1) We assigned unit costs using Canadian government estimates computed annually through established methodologies. Because physician payment mechanisms in Canada are heterogeneous, physician cost estimates are less reliable than those for inpatient costs [13]. We repeated all analyses using an alternative data source representing billable fees per procedure, which provides a lower bound for physician costs and conservatively estimates cost savings. (2) The economic evaluation used a linear model to study mean costs and effects. This enabled us to address the key policymaking question of interest to decision makers, which concerns the absolute change in effects per change in costs conferred by the intervention. Linear regression models may result in incorrect standard errors. While our bivariate multilevel linear models account for clustering and bivariate correlations at hospital and individual levels, the use of Markov chain Monte Carlo methods to generate joint posterior distributions of incremental costs and effects yielded sample means and estimates of uncertainty robust to skewed costs $[17,28]$. The main trial analysis appropriately modelled caesarean sections as binary, and its results, presented on the odds ratio scale, should be considered the definitive estimates of intervention impact [6]. (3) The central limit theorem, upon which valid inference of sample means is based, may not hold with small sample sizes [29]. Our dataset has 105,351 patients from 32 independent clusters with a median cluster size of 2644 patients (range: 638 to 9608). (4) Sample size calculations for the QUARISMA trial were not designed to test costeffectiveness hypotheses. Methodological guidance suggests that economic evaluation should focus on characterizing differences between study group, rather than formal hypothesis testing [21]. This trial had a large sample size, and overall and stratified cost-effectiveness analyses were able to detect a difference between study groups at a significance level of 0.05 , suggesting that the sample size was adequate. (5) Our analysis was based on a health system perspective. Patients and providers may have personal preferences for caesarean sections, positive or negative, not 
captured by this analysis. (6) The main trial analysis identified a possible random increase in complications in the control group. Some proportion of the cost savings conferred by the intervention might be attributable to random variation; this cannot be ascertained from the trial data. (7) Hospitals eligible for inclusion in this trial had a minimum annual delivery volume of 300; intervention performance in smaller hospitals is unknown, and this may affect the scope for achieving benefits at scale. Returns from the QUARISMA trial itself may range from $\$ 0.5$ million to $\$ 5$ million in the 1-year post-intervention period alone (Additional file 1: Table S10). (8) This study was done in a single jurisdiction; differences in health system organization and financing may limit transferability of results to other settings. A national budget impact analysis is being conducted to translate these results for all Canadian provinces. (9) The relatively short trial time horizon does not elucidate how the intervention impact may evolve over time.

A similar program could be beneficial in other regions with similar or higher caesarean section rates [6]. Costeffectiveness results can be adapted to other jurisdictions by adjusting for local baseline caesarean rates, health system costs, and proportions of high- and low-risk patients.

\section{Conclusions}

From a healthcare payer perspective, a multifaceted intervention involving audits and feedback resulted in a small reduction in caesarean deliveries and an important reduction in per-patient costs. These results were achieved without increasing neonatal and maternal morbidity and mortality.

In keeping with the theory of change for the QUARISMA intervention, which attempted to optimize medical practice by reducing unnecessary caesareans, our study found that women with low-risk pregnancies experienced statistically significant reductions in caesarean sections and costs, while changes for the high-risk subgroup were not significant. We also found evidence of improved quality along the continuum of care. Cost reductions were driven principally by lower rates of neonatal complications and corresponding lower use of resources within the intervention group. These changes are consistent with improvements in the quality of care in intervention group hospitals.

Findings from our study provide critical new evidence concerning a safe and possibly sustainable strategy to reduce unnecessary caesarean sections and shed new light on the potential for audit and feedback interventions to improve quality of care while controlling costs. Delivery of the intervention at wider scale accompanied by further research is required to assess whether the improvements in clinical practice driving reductions in caesarean sections and cost savings are sustainable over time.

\section{Additional file}

Additional file 1: Supplementary material for a cluster-randomized trial to reduce caesarean delivery rates in Quebec: cost-effectiveness analysis. (PDF $716 \mathrm{~kb}$ )

\section{Abbreviations}

CIHI: Canadian Institute for Health Information; CS: Caesarean section; ICER: Incremental cost-effectiveness ratio; NPD: National Physician Database; PCE: Patient Cost Estimator; QUARISMA: Quality of Care, Obstetrics Risk Management, and Mode of Delivery (trial)

\section{Acknowledgements}

The authors gratefully acknowledge Drs. François Audibert, Anne-Monique Nuyt, Jean-Charles Pasquier, and Annie Li for expert guidance on care pathways and costs for maternal and neonatal complications, and the editor and reviewers for thoughtful guidance that has improved the scientific quality of the paper.

\section{Funding}

The Canadian Institutes of Health Research (grants 200702MCT-171307-RFACFCF-153236 and MOP 81275) funded this study. The funding sources played no role in the study design, data collection, analysis and interpretation, writing of the report, or the decision to submit the paper for publication.

\section{Availability of data and materials}

The data that support the findings of this study are available on reasonable request from the senior author (NC), subject to approval of the local institutional review boards. The data are not publicly available because they contain information that could compromise privacy and consent for participating centres and individuals.

\section{Authors' contributions}

MJ, JSH, and NC conceived of the idea for the study and developed the study design. TD and NC provided the data. Statistical analysis was lead by ESWN in collaboration with MJ and CBT; all authors contributed to interpretation of results. MJ drafted the manuscript, and ESWN, CBT, JSH, TD, and $\mathrm{NC}$ revised it for important intellectual content. All authors read and approved the final manuscript. All authors agree to be accountable for all aspects of the work in ensuring that questions related to the accuracy or integrity of any part of the work are appropriately investigated and resolved.

\section{Competing interests}

The authors declare that they have no competing interests.

\section{Consent for publication}

Not applicable.

\section{Ethics approval and consent to participate}

The economic evaluation plan was pre-specified in the trial protocol and updated to reflect recent advances in analytic methods and reporting. On behalf of the 32 participating hospital centres, the following 32 local institutional review boards granted research ethics approval for the QUARISMA trial: (1) Comité d'éthique de la recherche du Centre intégré de santé et de services sociaux du Bas-Saint-Laurent; (2) Comité d'éthique de la recherche du Centre intégré universitaire de santé et de services sociaux du Saguenay-Lac-Saint-Jean (Hôtel-Dieu de Roberval); (3) Directeur des services professionnels du Hôtel-Dieu d'Alma; (4) Comité d'éthique de la recherche, Centre de santé et de services sociaux de Chicoutimi; (5) Comité d'éthique de la recherche du Centre hospitalier universitaire de Québec - Université Laval; (6) Comité d'éthique mixte du Centre Hospitalier du Centre-de-laMauricie; (7) Comité d'éthique de la recherche multi-clientèle du Centre intégré universitaire de santé et de services sociaux de la Mauricie-et-duCentre-du-Québec; (8) Comité d'éthique de la recherche du Centre Hospitalier Universitaire de Sherbrooke; (9) Comité d'éthique de la recherche Centre Hospitalier de St. Mary; (10) Directeur des services professionnels, Hôpital général du Lakeshore; (11) Directeur des services professionnels, Hôpital St-Luc (Centre hospitalier de I'Université de Montréal); (12) Comité d'éthique de la recherche du Centre hospitalier universitaire Sainte-Justine; (13) Comité d'éthique de la recherche du Centre intégré universitaire de santé et de services sociaux de l'Est-de-l'̂lle-de-Montréal (Hôpital Maisonneuve-Rosemont); (14) 
Directeur des services professionnels, Centre hospitalier de Val d'Or; (15) Centre de santé et de services sociaux les Eskers de l'Abitibi; (16) Directeur des services professionnels, Centre hospitalier Rouyn-Noranda; (17) Directeur des services professionnels, Centre hospitalier régional de Sept-îles; (18) Centre de santé et services de Manicougan (Centre hospitalier régional Baie-Comeau); (19) Comité de bioéthique, Hôtel-Dieu de Lévis; (20) Centre hospitalier BeauceEtchemin; (21) Comité d'éthique de la recherche, Centre de santé et de services sociaux de Laval, Hôpital de la Cité-de-la-Santé; (22) Directeur des services professionnels, Centre hospitalier régional de Lanaudière; (23) Comité d'éthique de la recherche, Centre hospitalier Pierre-Le Gardeur; (24) Directeur des services professionnels, Centre hospitalier Mont-Laurier; (25) Directeur des services professionnels, Centre hospitalier St-Eustache; (26) Comité d'éthique de la recherche, Centre de santé et de services sociaux de Saint-Jérôme; (27) Directeur des services professionnels, Hôpital Brôme-Missisquoi-Perkins; (28) Directeur des services professionnels, Centre hospitalier Pierre-Boucher; (29) Directeur des services professionnels, Centre de santé et de services sociaux Richelieu-Yamaska (Hôpital Honoré-Mercier); (30) Comité d'éthique, Centre de santé et de services sociaux de la Haute-Yamaska (Centre hospitalier de Granby); (31) Comité d'éthique de la recherche, Hôtel-Dieu de Sorel; (32) Comité d'éthique du Centre de santé et de services sociaux Drummond (Hôpital Ste. Croix).

\section{Publisher's Note}

Springer Nature remains neutral with regard to jurisdictional claims in published maps and institutional affiliations.

\begin{abstract}
Author details
${ }^{1}$ Centre de Recherche du Centre Hospitalier de I'Université de Montréal (CRCHUM), Tour Saint-Antoine, Porte S03-910, 850, rue St-Denis, Montréal, Québec H2X OA9, Canada. ²Department of Health Management, Evaluation and Policy, School of Public Health, University of Montreal, Montréal, Québec, Canada. ${ }^{3}$ Department of Maternal, Neonatal, Child and Adolescent Health, World Health Organization, Geneva, Switzerland. ${ }^{4}$ Director's Office, London School of Hygiene and Tropical Medicine (LSHTM), London, UK. ${ }^{5}$ Department of Obstetrics and Gynecology, Centre Hospitalier Universitaire (CHU) de Sherbrooke, Sherbrooke, Québec, Canada. ${ }^{6}$ Andalusian School of Public Health, Granada, Spain. ${ }^{7}$ CIBER Epidemiologia y Salud Publica (CIBERESP), Instituto de Salud Carlos III, Madrid, Spain. ${ }^{8}$ Institute of Health Policy, Management and Evaluation, University of Toronto, Toronto, Ontario, Canada. ${ }^{9}$ Centre for Excellence in Economic Analysis and Research (CLEAR), Li Ka Shing Knowledge Institute, St Michael's Hospital, Toronto, Ontario, Canada. ${ }^{10}$ Department of Public Health Sciences, University of California, Davis, California, USA. ${ }^{11}$ Department of Biostatistics, Centre hospitalier universitaire (CHU) Sainte-Justine, Université de Montréal, Montréal, Québec, Canada. ${ }^{12}$ Département Obstétrique et Gynécologie, Centre Hospitalier de I'Université Laval (CHUL), Québec, Québec, Canada.
\end{abstract}

\section{Received: 11 December 2016 Accepted: 20 April 2017} Published online: 22 May 2017

\section{References}

1. Betran AP, Ye J, Moller AB, Zhang J, Gulmezoglu AM, Torloni MR. The increasing trend in caesarean section rates: global, regional and national estimates: 1990-2014. PLoS One. 2016;11(2):E0148343.

2. Betran AP, Torloni MR, Zhang J, Ye J, Mikolajczyk R, Deneux-Tharaux C, Oladapo OT, Souza JP, Tuncalp O, Vogel JP, et al. What is the optimal rate of caesarean section at population level? A systematic review of ecologic studies. Reprod Health. 2015;12:57.

3. Betran AP, Torloni MR, Zhang JJ, Gulmezoglu AM, WHO Working Group On Caesarean Section. WHO statement on caesarean section rates. BJOG. 2015; 23(45):149-50.

4. Villar J, Valladares E, Wojdyla D, Zavaleta N, Carroli G, Velazco A, Shah A, Campodonico L, Bataglia V, Faundes A, et al. Caesarean delivery rates and pregnancy outcomes: the 2005 WHO global survey on maternal and perinatal health in Latin America. Lancet. 2006;367(9525):1819-29.

5. Khunpradit S, Tavender E, Lumbiganon P, Laopaiboon M, Wasiak J, Gruen R. Non-clinical interventions for reducing unnecessary caesarean section. Cochrane Database Syst Rev. 2011;6:Cd005528.

6. Chaillet N, Dumont A, Abrahamowicz M, Pasquier JC, Audibert F, Monnier P, Abenhaim HA, Dube E, Dugas M, Burne R, et al. A clusterrandomized trial to reduce cesarean delivery rates in Quebec. N Engl J Med. 2015;372(18):1710-21.
7. Statistics Canada. Life expectancy, at birth and at age 65, by sex, Canada, provinces and territories, annual (years) (CANSIM Table 102-0512). Ottawa: Statistics Canada; 2012.

8. Institut de la statistique du Québec. Profils economiques par pays et territoire, et tableaux comparatifs par indicateur, 2005-2014. Québec: Institut de la statistique du Québec; 2015.

9. Canadian Agency for Drugs and Technologies in Health (CADTH). Guidelines for the economic evaluation of health technologies. Ottawa: Canadian Agency for Drugs and Technologies in Health (CADTH); 2006.

10. Ramsey SD, Willke RJ, Glick H, Reed SD, Augustovski F, Jonsson B, Briggs A, Sullivan SD. Cost-effectiveness analysis alongside clinical trials II-An ISPOR Good Research Practices Task Force Report. Value Health. 2015;18(2):161-72.

11. Statistics Canada. Births, estimates, by province and territory. (CANSIM Table 051-0004). 2016. Ottawa.

12. Canadian Institute for Health Information $(\mathrm{CIHI})$. Patient cost estimator methodology notes and glossary. Ottawa: $\mathrm{ClHI}$; 2015. p. 9.

13. Canadian Institute for Health Information (CIHI). National Physician Database 2011-2012 Data Release. Ottawa: CIHI; 2013.

14. Official Exchange Rate (LCU per US\$, Period Average). 2013. http://data. worldbank.org/indicator/PA.NUS.FCRF?locations=CA. Accessed 30 Oct 2015.

15. Khandker SR, Koolwal GB, Hussain AS. Handbook on impact evaluation: quantitative methods and practices. Washington, DC: The World Bank; 2010.

16. Dumont A, Fournier P, Abrahamowicz M, Traore M, Haddad S, Fraser WD, for the QUARITE research group. Quality of care, risk management, and technology in obstetrics to reduce hospital-based maternal mortality in Senegal and Mali (QUARITE): a cluster-randomised trial. Lancet. 2013; 382(9887):146-57.

17. Gomes M, Ng ES, Grieve R, Nixon R, Carpenter J, Thompson SG. Developing appropriate methods for cost-effectiveness analysis of cluster randomized trials. Med Decis Making. 2012;32(2):350-61.

18. Ng ES-W, Diaz-Ordaz K, Grieve R, Nixon RM, Thompson SG, Carpenter JR. Multilevel models for cost-effectiveness analyses that use cluster randomised trial data: an approach to model choice. Stat Methods in Med Res. 2013. doi: 10.1177/0962280213511719

19. Rasbash J, Charlton C, Browne WJ, Healy M, Cameron B. MLwiN Version 2.1. In: Centre for Multilevel Modelling. Bristol: University of Bristol; 2009.

20. Leckie G, Charlton C. runmlwin: a program to run the MLwiN multilevel modeling software from within Stata. J Stat Software. 2013;52(11):40.

21. O'Brien BJ, Briggs AH. Analysis of uncertainty in health care costeffectiveness studies: an introduction to statistical issues and methods. Stat Methods Med Res. 2002;11(6):455-68.

22. Browne WJ. MCMC estimation in MLwiN, V2.26. Bristol: Centre For Multilevel Modelling. Bristol: University Of Bristol; 2012.

23. Régie de L'assurance maladie du Québec. Manuel de Facturation: Rémunération a L'acte (Médecins Spécialistes), Maj 85 Edn; 2013

24. Ivers N, Jamtvedt G, Flottorp S, Young JM, Odgaard-Jensen J, French SD, O'Brien MA, Johansen M, Grimshaw J, Oxman AD. Audit and feedback: effects on professional practice and healthcare outcomes. Cochrane Database Syst Rev. 2012;6:Cd000259.

25. Fretheim A, Aaserud M, Oxman AD. Rational Prescribing in Primary Care (RaPP): economic evaluation of an intervention to improve professional practice. PLoS Med. 2006;3(6):E216.

26. Montgomery AA, Emmett CL, Fahey T, Jones C, Ricketts I, Patel RR, Peters TJ, Murphy DJ, Di ASG. Two decision aids for mode of delivery among women with previous caesarean section: randomised controlled trial. BMJ. 2007; 334(7607):1305.

27. Hollinghurst S, Emmett C, Peters TJ, Watson H, Fahey T, Murphy DJ, Montgomery A. Economic evaluation of the DIAMOND randomized trial: cost and outcomes of 2 decision aids for mode of delivery among women with a previous cesarean section. Med Decis Making. 2010;30(4):453-63.

28. Briggs A, Nixon R, Dixon S, Thompson S. Parametric modelling of cost data: some simulation evidence. Health Econ. 2005;14(4):421-8.

29. Nixon RM, Wonderling D, Grieve RD. Non-parametric methods for costeffectiveness analysis: the central limit theorem and the bootstrap compared. Health Econ. 2010;19(3):316-33.

30. Exchange rates. 2013. http://www.bankofcanada.ca/rates/exchange/legacynoon-and-closing-rates/. Accessed 30 Oct 2015. 